\title{
A MULTI-LINK COMMUNICATION CONNECTIVITY GAME UNDER HOSTILE INTERFERENCE
}

\author{
Andrey Garnaev ${ }^{1}$, Wade Trappe ${ }^{1}$, Narayan B. Mandayam ${ }^{2}$, H.Vincent Poor ${ }^{3}$ \\ ${ }^{1}$ WINLAB, Rutgers University, North Brunswick, NJ, USA (e-mail: andrey.garmaev@gmail.com, \\ trappe@winlab.rutgers.edu), ${ }^{2}$ Department of Electrical and Computer Engineering, Rutgers University, Piscataway, NJ, \\ USA, (e-mail: narayan@winlab.rutgers.edu), ${ }^{3}$ Department of Electrical Engineering, Princeton University, Princeton, NJ \\ USA (e-mail: poor@princeton.edu)
}

NOTE: Corresponding author: A. Garnaev, e-mail: andrey.garmaev@gmail.com

\begin{abstract}
In this paper, we consider a communication connectivity problem involving a primary user (transmitter, for example, a Ground Control Station (GCS)) servicing a group of secondary users (receivers, for example, drones) under hostile interference. We formulate this multi-link communication connectivity problem, where the channels are affected by Rayleigh fading, as a zero-sum power resource allocation game between a transmitter and an adversary (jammer). The transmitter's objective is to maximize the probability of communication connectivity with all the receivers. It is proven that the problem has unique equilibrium in power allocation strategies, and the equilibrium is derived in closed form. Moreover, we reduce the problem of designing the equilibrium in power resource allocation strategies to the problem of finding a fixed point of a real-valued function. An algorithm based on the bisection method to find the fixed point (and so equilibrium strategies) is developed, and its convergence is proven.
\end{abstract}

Keywords - communication connectivity, interference, jamming, Nash equilibrium, resource allocation

\section{INTRODUCTION}

Communication between a transmitter and a receiver under hostile interference is a well-studied problem in the wireless literature (see, for example, the survey in [1]). Such problems are multi-objective problems since they deal with different agents (say, a transmitter and an adversary (jammer)), and each of these agents has its own objective. Game theory supplies concepts for analyzing and solving such multi-objective problems [2], and, thus, game theory has been widely used to model jamming problems. Typically, jamming problems can be categorized according to two frameworks: (i) maintaining communication reliability and (ii) maintaining communication connectivity. In communication reliability problems, the transmitter's payoff is a function of throughput or Signal-to-Interference-plus-Noise Ratio (SINR) at the receiver, and the transmitter intends to maximize such payoff $[3,4,5,6,7,8,9,10,11]$. Meanwhile, in communication connectivity problems the transmitter must keep its SINR greater than or equal to a threshold value to ensure a connection can be sustained $[12,13,14,15,16,17$, $18,19]$. In this paper, we consider the communication connectivity problem under hostile interference involving a transmitter servicing a group of secondary users (receivers) with the channels affected by Rayleigh fading. We formulate and solve this Multi-Link Communication Connectivity (MLCC) problem as a zero-sum power allocation problem between the transmitter and the jammer, where the transmitter wants to maximize the Probability of Communication Connectivity (PCC) with all the receivers.

One of the core difficulties in communication connectivity problems is that the transmitter has to keep its SINR of the transmitted signal at the receiver greater or equal to a threshold value, which we refer to as the Threshold of Communication Connectivity (TCC). This leads generally to to the non-existence of equilibria in power-level assignment strategies, i.e., pure strategies, even in a Single-Link Communication Connectivity (SLCC) problem [14, 17], and it may cause destabilization of communication. One of the ways to stabilize such systems, i.e., to make them have equilibrium, is to extend the set of feasible strategies to mixed strategies (in other words, to assign the equilibrium strategy via randomization (lottery) over pure strategies). Although using a lottery introduces a factor of uncertainty for the decision maker it allows it to find an equilibrium in such mixed strategies for the MLCC problem via the Colonel Blotto game approach $[12,13,16,18$, $19,17]$ and for the SLCC problem via a war of attrition game approach [17].

To avoid introducing a random factor in decision making, in $[14,15]$, another approach was suggested to stabilize such communication. In $[14,15]$, it was proven that if channels are affected by Rayleigh fading and background noise at the receiver is negligible, then an equilibrium in power-level assignment strategies (i.e., pure strategies) exists and is unique for an SLCC problem.

The goal of this paper is to prove that if channels are affected by Rayleigh fading and any background noise, a random factor can be eliminated in decision making for communication stabilization even in MLCC systems. To the best of the authors' knowledge this problem has not been considered before.

The main contributions of this paper are as follows:

(1) A problem of multi-link communication connectivity 
under jamming of a transmitter with a group of receivers when the channels are affected by Rayleigh fading is modeled as a zero-sum power power allocation game.

(2) Existence and uniqueness of the equilibrium in power allocation strategies are proven. Thus, in contrast to Colonel Blotto games, if channels are affected by Rayleigh fading, then stability of communication connectivity in a multi-link system can be maintained without introducing a random factor for a decision maker.

(3) We reduce the problem of finding the equilibrium in power resource allocation strategies to the problem of solving a fixed point equation in a scalar variable. An algorithm based on the bisection method to find the fixed point (and so equilibrium strategies) is developed, and its convergence is proven. This algorithm can be considered to be a learning algorithm since it allows one to reduce the zone of uncertainty for the equilibrium by a factor of two on iteration.

The organization of this paper is as follows. In Section 2, a short summary of an SLCC model is given. In Section 3, its generalization for the MLCC scenario is suggested as a zero-sum game between the transmitter communicating with a group of receivers and the jammer. In Section 4, the equilibrium strategies are designed and the uniqueness of the equilibrium is proven. In Section 5, the equilibrium strategies are established in closed form for the boundary cases of network parameters: (a) for small or large TCC, (b) for small or large total jamming and transmission power budgets and (c) for small background noise at the receivers. In Section 6, an algorithm to design the equilibrium in the general case is presented and its convergence is proven. Finally, in Section 7, illustrations of the results are given, and, in Section 8, conclusions are offered. All proofs are provided in the appendix.

\section{SHORT OVERVIEW OF A SINGLE-LINK COMMUNICATION MODEL}

In this section following $[14,15]$ we give a short overview of the SLCC model with one transmitter communicating directly to a single receiver. This communication is affected by hostile interference from a jammer. Let $G$ and $H$ be the channel power gains from the transmitter to the receiver and the jammer to the receiver, respectively. In practice, the channel power fading gains depend on distances, fading and antenna characteristics. In general, the channel power gains are random variables (e.g., representing channel fading) with means $\boldsymbol{E}[G]=g$ and $\boldsymbol{E}[H]=$ $h$. Let $p$ and $q$ be the power levels used by the transmitter and the jammer, respectively. Thus, $\mathbb{R}_{+}$is the set of feasible strategies for the transmitter as well as for the jammer. The receiver also is affected by noise power $N$. Thus, the SINR at the receiver is given by

$$
\operatorname{SINR}(p, q)=\frac{H p}{N+G q}
$$

We say that the communication from the transmitter to the receiver is maintained if and only if the SINR at the receiver is greater than or equal to a given TCC $\epsilon$, i.e., the following condition holds:

$$
\operatorname{SINR}(p, q) \geq \epsilon
$$

This TCC $\epsilon$ depends on the system's requirements, such as bit rate and bit error rate (BER).

Then, since $G$ and $H$ are random variables, the probability that the link between the transmitter and the receiver is maintained, i.e., PCC, is given by

$$
\boldsymbol{P}(p, q)=\boldsymbol{P}(\operatorname{SINR}(p, q) \geq \epsilon) .
$$

For the case when the channels are affected by Rayleigh fading (i.e., $G$ and $H$ are exponential random variables with means $\boldsymbol{E}[G]=g$ and $\boldsymbol{E}[H]=h$, respectively), by $[14,15]$, the probability (3) can be represented as follows:

$$
P(p, q)=\frac{e^{-\epsilon \frac{N}{h p}}}{1+\epsilon \frac{g q}{h p}} .
$$

Note that $\boldsymbol{P}(p, q)$ is continuous in $p \geq 0$ and $q \geq 0$ and $\boldsymbol{P}(0, q)=0$ for $q \geq 0$.

\section{A MULTI-LINK COMMUNICATION CON- NECTIVITY MODEL}

In this section, we generalize the SLCC problem to the MLCC problem involving a transmitter with $n$ receivers as follows:

- We assume that the transmitter is equipped with $n$ directed antennas to communicate with $n$ receivers.

- Let $p=\left(p_{1}, \ldots, p_{n}\right)$ be the strategy of the transmitter where $p_{i}$ is the power assigned to communicate with the receiver $i$ and

$$
\sum_{i \in \mathcal{N}} p_{i}=\bar{P} \text { and } p_{i} \geq 0, i \in \mathcal{N}
$$

with $\bar{P}$ is the transmitter's total power budget and $\mathcal{N} \triangleq\{1, \ldots, n\}$. Denote by $\mathcal{P}$ the set of all feasible strategies for the transmitter.

- Let the jammer also be equipped with $n$ directed antennas.

- Let $q=\left(q_{1}, \ldots, q_{n}\right)$ be strategy of the jammer, where $q_{i}$ is the power assigned to jam the communication between the transmitter with receiver $i$, and

$$
\sum_{i \in \mathcal{N}} q_{i}=\bar{Q} \text { and } q_{i} \geq 0, i \in \mathcal{N}
$$

with $\bar{Q}$ is the total jamming power budget. Denote by $\mathcal{Q}$ the set of all feasible strategies for the jammer. 
- By (4), the PCC with the receiver $i$ is given as follows:

$$
\boldsymbol{P}_{i}\left(p_{i}, q_{i}\right)=\frac{e^{-\epsilon \frac{N_{i}}{h_{i} p_{i}}}}{1+\epsilon \frac{g_{i} q_{i}}{h_{i} p_{i}}},
$$

where $h_{i}$ and $g_{i}$ are channel gains and $N_{i}$ is the background noise.

- The PCC with all the $n$ receivers is given as follows:

$$
\pi(p, q)=\prod_{i \in \mathcal{N}} P_{i}\left(p_{i}, q_{i}\right)=\prod_{i \in \mathcal{N}} \frac{e^{-\epsilon \frac{N_{i}}{h_{i} p_{i}}}}{1+\epsilon \frac{g_{i} q_{i}}{h_{i} p_{i}}} .
$$

The goal of the transmitter is to maximize this PCC, while the jammer wants to minimize this probability. Such a problem could arise in military operations where one radio station (say, GCS) must transmit data to $n$ military units (e.g., drones) under hostile interference. Thus, $\pi(p, q)$ is the payoff to the transmitter, while for the jammer $\pi(p, q)$ is the cost function. Thus, here we deal with a zero sum game. We look for the Nash equilibrium [2]. Recall that $\left(p_{*}, q_{*}\right)$ is a Nash equilibrium in a zero-sum game if and only if the following inequalities hold:

$$
\pi\left(p, q_{*}\right) \leq \pi\left(p_{*}, q_{*}\right) \leq \pi\left(p_{*}, q\right) \text { for all }(p, q) \in \mathcal{P} \times \mathcal{Q} .
$$

Let

$$
\begin{aligned}
v(p, q) & =\ln (\pi(p, q)) \\
& =\sum_{i \in \mathcal{N}} \ln \left(\frac{h_{i} p_{i}}{h_{i} p_{i}+\epsilon g_{i} q_{i}}\right)-\epsilon \sum_{i \in \mathcal{N}} \frac{N_{i}}{h_{i} p_{i}} .
\end{aligned}
$$

Since $\ln (\cdot)$ is an increasing function, the problem to find the Nash equilibrium with payoff $\pi(p, q)$ to the transmitter is equivalent to finding the Nash equilibrium with payoff $v(p, q)$ to the transmitter, i.e., $\operatorname{such}\left(p_{*}, q_{*}\right)$ that

$$
v\left(p, q_{*}\right) \leq v\left(p_{*}, q_{*}\right) \leq v\left(p_{*}, q\right) \text { for all }(p, q) \in \mathcal{P} \times \mathcal{Q} .
$$

Denote this game by $\Gamma=\Gamma(v, \mathcal{P}, Q)$.

Note that the transmitter's equilibrium strategy also reflects the most fair power resource allocation to maintain communication with all the receivers under the worst hostile interference since the utility $v$ given by (10) can also be considered as a proportional fairness utility [20, 21].

Theorem 1 The game $\Gamma(v, \mathcal{P}, \mathcal{Q})$ has at least one Nash equilibrium.

The proof can be found in Appendix 9.1.

Note that, generally in resource allocation problems even if the payoffs are concave the game might have multiple equilibria (see, for example, [22]). In this paper we establish uniqueness of the equilibrium as a side effect of solving the best response equations associated with (11).

\section{SOLUTION OF THE GAME}

In this section we find equilibrium strategies in closed form using a constructive approach via finding all solutions of the best response equations. Recall that, by (11), $(p, q)$ is a Nash equilibrium if and only if each of these strategies is the best response to the other, i.e., $(p, q)$ is a solution of the best response equations:

$$
\begin{aligned}
& p=\operatorname{argmax}\{v(p, q): p \in \mathcal{P}\}, \\
& q=\operatorname{argmin}\{v(p, q): q \in \mathcal{Q}\} .
\end{aligned}
$$

Note that (12) and (13) are Non-Linear Programming (NLP) problems.

\subsection{Explicit form for the equilibrium strategies}

In this section we find in closed form all of the possible solutions of the best response equations, i.e., equilibrium strategies, as functions of two auxiliary parameters $\omega$ and $v$ (Lagrange multipliers for the NLP problems (12) and (13) correspondingly).

Proposition 1 Each equilibrium $(p, q)$ of the game $\Gamma(v, \mathcal{P}, \mathcal{Q})$ has to have the following form

$$
\begin{aligned}
& p_{i}=p_{i}(\omega, v) \\
& \triangleq \begin{cases}\sqrt{\frac{\epsilon N_{i}}{h_{i} \omega}}, & \frac{\epsilon g_{i}^{2}}{N_{i} h_{i}} \omega \leq v^{2}, \\
\frac{1+\sqrt{1+4 \frac{\epsilon N_{i}}{h_{i}}\left(\omega+\frac{h_{i}}{\epsilon g_{i}} v\right)}}{2\left(\omega+\frac{h_{i}}{\epsilon g_{i}} v\right)}, & \frac{\epsilon g_{i}^{2}}{N_{i} h_{i}} \omega>v^{2}\end{cases}
\end{aligned}
$$

and

$$
\begin{aligned}
q_{i} & =q_{i}(\omega, v) \\
& \triangleq\left[\frac{1}{v}-\frac{h_{i}}{\epsilon g_{i}} \frac{1+\sqrt{1+4 \frac{\epsilon N_{i}}{h_{i}}\left(\omega+\frac{h_{i}}{\epsilon g_{i}} v\right)}}{2\left(\omega+\frac{h_{i}}{\epsilon g_{i}} v\right)}\right\rfloor_{+},
\end{aligned}
$$

where $i \in \mathcal{N}$ and $[\xi\rfloor_{+} \triangleq \max \{\xi, 0\}$. Thus, $\lfloor\xi\rfloor_{+}=\xi$ if $\xi \geq 0$ and $[\xi\rfloor_{+}=0$ otherwise. Moreover, $\omega>0$ and $v>0$ are solutions of the following equations:

$$
\begin{aligned}
& P(\omega, v) \triangleq \sum_{i \in \mathcal{N}} p_{i}(\omega, v)=\bar{P} \\
& Q(\omega, v) \triangleq \sum_{i \in \mathcal{N}} q_{i}(\omega, v)=\bar{Q} .
\end{aligned}
$$

The proof can be found in Appendix 9.2.

Note that, by Theorem 1 and Proposition 1, the non-linear equations (16) and (17) have at least one solution. To find this solution and to establish its uniqueness we cannot 
apply a non-linear modification for the Gaussian elimination method suggested in [23] of a search game, and, further, in [24], applied for an Orthogonal FrequencyDivision Multiplexing (OFDM) jamming game and, in [22], for a multi-user OFDM game. The issue is that although we can establish monotonicity in one direction by both variables for one of the functions (in our case, $P$ ), the other function (in our case, $Q$ ) generally is not monotonic in opposite directions of its variables. Instead of that approach, we reduce the non-linear equations (16) and (17) to a fixed point equation on $v$ and prove that it has a unique solution and then develop an algorithm to find this fixed point.

\subsection{Auxiliary monotonicity properties}

In this section we establish monotonicity properties of $P(\omega, v)$ with respect to its parameters and derive a bijective relation between $\omega$ and $v$ based on (16).

Proposition 2 Function $P(\omega, v)$ has the following properties:

(a) $P(\omega, v)$ is continuous in $\omega>0$ and $v \geq 0 ;^{1}$

(b) for a fixed $v>0, P(\omega, v)$ is decreasing in $\omega$ from infinity for $\omega \downarrow 0$ to zero for $\omega \uparrow \infty$;

(c) for a fixed $\omega>0, P(\omega, v)$ is decreasing in $v$ from

$$
P(\omega, 0)=\frac{1}{2 \omega} \sum_{i \in \mathcal{N}}\left(1+\sqrt{1+4 \frac{\epsilon N_{i}}{h_{i}} \omega}\right) \text { for } v=0
$$

to

$$
P(\omega, \infty)=\sum_{i \in \mathcal{N}} \sqrt{\frac{\epsilon N_{i}}{h_{i} \omega}} \text { for } v \uparrow \infty
$$

(d) for a fixed $v \geq 0$ there exists the unique $\omega=\Omega(v)$ such that

$$
P(\Omega(v), v)=\bar{P} .
$$

Moreover, $\Omega(v) \in\left[\Omega_{\infty}, \Omega_{0}\right]$ and $\Omega(v)$ can be found via the bisection method;

(e) $\Omega(v)$ is continuous and decreasing from $\Omega_{0}$ for $v=0$ to $\Omega_{\infty}$ for $v \uparrow \infty$, where $\Omega_{0}$ is the unique root of the equation:

$$
\frac{1}{2 \Omega_{0}} \sum_{i \in \mathcal{N}}\left(1+\sqrt{1+4 \frac{\epsilon N_{i}}{h_{i}} \Omega_{0}}\right)=\bar{P}
$$

and

$$
\Omega_{\infty}=\frac{\epsilon}{\bar{P}^{2}}\left(\sum_{i \in \mathcal{N}} \sqrt{\frac{N_{i}}{h_{i}}}\right)^{2} .
$$

The proof can be found in Appendix 9.3.

\footnotetext{
$1 \xi \downarrow a$ denotes that $\xi$ tends to $a$ decreasingly. Similarly, $\xi \uparrow a$ denotes that $\xi$ tends to $a$ increasingly.
}

\subsection{An auxiliary fixed point equation}

In this section we reduce Equation (16) and Equation (17) to a fixed point equation. First note that multiplying both sides of Equation (17) by $v$ implies that Equation (17) is equivalent to

$$
\tilde{Q}(\omega, v)=\bar{Q} v
$$

where

$$
\tilde{Q}(\omega, v) \triangleq v Q(\omega, v) .
$$

Moreover, by (15) and (24), we have that

$\tilde{Q}(\omega, v) \triangleq \sum_{i \in \mathcal{N}}\left[1-\frac{\frac{h_{i}}{\epsilon g_{i}} v\left(1+\sqrt{1+4 \frac{\epsilon N_{i}}{h_{i}}\left(\omega+\frac{h_{i}}{\epsilon g_{i}} v\right)}\right)}{2\left(\omega+\frac{h_{i}}{\epsilon g_{i}} v\right)}\right]_{+}$

In the following proposition we establish monotonicity of $\tilde{Q}(\omega, v)$ on parameters $\omega$ and $v$, and reduce Equation (16) and Equation (17) to a fixed point equation.

Proposition 3 Function $\tilde{Q}(\omega, v)$ has the following properties:

(a) $\tilde{Q}(\omega, v)$ is continuous in $\omega>0$ and $v \geq 0$;

(b) for a fixed $\omega>0, \tilde{Q}(\omega, v)$ is decreasing in $v$ from $n$ for $v=0$ to zero for $v \geq \bar{v}$ where $\bar{v}$ is the unique positive root of the equation

$$
A+B \bar{v}=C \bar{v}^{3 / 2}
$$

with

$$
A=2 \Omega_{0}, B=2 \max _{i \in \mathcal{N}} \frac{h_{i}}{\epsilon g_{i}} \text { and } C=\min _{i \in \mathcal{N}} \frac{h_{i} N_{i}^{1 / 2}}{\epsilon g_{i}^{3 / 2}} ;
$$

(c) for a fixed $v>0, \tilde{Q}(\omega, v)$ is increasing in $\omega$ and tends to $n$ for $\omega \uparrow \infty$;

(d) function

$$
\varphi(v) \triangleq \tilde{Q}(\Omega(v), v)
$$

is decreasing in $v$ from $n$ for $v=0$ to zero for $v \geq \bar{v}$,

(e) The following fixed point equation has the unique positive root $v_{*}$ :

$$
\varphi\left(v_{*}\right) / \bar{Q}=v_{*} .
$$

This root can be found via the bisection method with $[0, \bar{v}]$ as the initial localization interval for such $v_{*}$.

The proof can be found in Appendix 9.4. 


\subsection{Equilibrium and its uniqueness}

In this section we find the equilibrium and establish its uniqueness.

Theorem 2 In the game $\Gamma(v, \mathcal{P}, \mathcal{Q})$, Nash equilibrium $(p, q)$ is unique. Moreover, this Nash equilibrium is $(p(\omega, v), q(\omega, v))$ given by (14) and (15), where $v=v_{*}$ uniquely given by (29) and $\omega=\Omega\left(v_{*}\right)$ uniquely given by (20).

The proof can be found in Appendix 9.5.

\section{THE BOUNDARY CASES}

In this section we find the equilibrium strategies in closed form for boundary cases of network parameters such as the TCC, the total jamming/transmission power resources and background noise at the receivers.

\subsection{Negligible background noise at the re-} ceivers

In this section we consider the scenario with negligible background noise at the receivers.

Proposition 4 Let the background noise at the receivers be negligible, i.e.,

$$
N_{i}=0 \text { for } i \in \mathcal{N} \text {. }
$$

Then the unique Nash equilibrium $(p, q)$ is given as follows:

$$
\begin{aligned}
p_{i} & =\frac{g_{i} \bar{Q} /\left(\epsilon g_{i} \bar{Q}+h_{i} \bar{P}\right)}{\sum_{j \in \mathcal{N}} g_{j} \bar{Q} /\left(\epsilon g_{j} \bar{Q}+h_{j} \bar{P}\right)} \bar{P}, \\
q_{i} & =\frac{g_{i} \bar{Q} /\left(\epsilon g_{i} \bar{Q}+h_{i} \bar{P}\right)}{\sum_{j \in \mathcal{N}} g_{j} \bar{Q} /\left(\epsilon g_{j} \bar{Q}+h_{j} \bar{P}\right)} \bar{Q}, \quad i \in \mathcal{N} .
\end{aligned}
$$

The proof can be found in Appendix 9.6.

Proposition 4 implies that, for negligible background noise at the receivers, the equilibrium strategies of the transmitter and the jammer are proportional to ratio $\bar{P} / \bar{Q}$. Note that, in the SLCC problem solved in [14, 15] for negligible background noise at the receiver, equilibrium strategies are given in closed form. Proposition 4 also supplies the equilibrium strategies in closed form for the MLCC problem. Thus, an increase in the number of communication links does not lead to an increase in the complexity involved in designing the equilibrium strategies.

\subsection{Large and small total transmission power}

In this section we consider the cases where total transmission power is either large or small.

Proposition 5 Let the total transmission power $\bar{P}$ be large. Then the Nash equilibrium $(p, q)$ can be approxi- mated as follows:

$$
\begin{aligned}
q_{i} & \approx \frac{g_{i}}{h_{i}}\left[\tau-\frac{N_{i} h_{i}}{g_{i}^{2}}\right]_{+}, \\
p_{i} & \approx \frac{\bar{P}}{T} \begin{cases}\sqrt{\frac{N_{i}}{h_{i}}}, & \tau \leq \frac{N_{i} h_{i}}{g_{i}^{2}}, \\
\sqrt{\tau} \frac{g_{i}}{h_{i}}, & \tau>\frac{N_{i} h_{i}}{g_{i}^{2}},\end{cases}
\end{aligned}
$$

where $\tau$ is the unique positive root of the equation:

$$
\sum_{i \in \mathcal{N}} \frac{g_{i}}{h_{i}}\left\lfloor\tau-\frac{N_{i} h_{i}}{g_{i}^{2}}\right\rfloor_{+}=\bar{Q}
$$

and

$$
T=\sum_{\tau \leq N_{i} h_{i} / g_{i}^{2}} \sqrt{\frac{N_{i}}{h_{i}}}+\sqrt{\tau} \sum_{\tau>N_{i} h_{i} / g_{i}^{2}} \frac{g_{i}}{h_{i}} .
$$

The proof can be found in Appendix 9.7.

Proposition 6 Let the total transmission power $\bar{P}$ be small. Then the Nash equilibrium $(p, q)$ can be approximated as follows:

$$
\begin{aligned}
& p_{i} \approx \frac{\sqrt{N_{i} / h_{i}}}{\sum_{j \in \mathcal{N}} \sqrt{N_{j} / h_{j}}} \bar{P}, \\
& q_{i} \approx \bar{Q} / n \text { for } i \in \mathcal{N} .
\end{aligned}
$$

The proof can be found in Appendix 9.8.

In particular, Proposition 5 and Proposition 6 imply that for large or small total transmission power the transmitter's strategies and the jammer strategy are insensitive to the TCC.

\subsection{Large or small total jamming power}

In this section we consider the cases where the total jamming power is either large or small.

Proposition 7 Let the total jamming power $\bar{Q}$ be large. Then, the Nash equilibrium $(p, q)$ can be approximated as follows:

$$
\begin{aligned}
& p_{i} \approx p_{i}\left(\Omega_{0}, 0\right)=\frac{1}{2 \Omega_{0}} \sum_{i \in \mathcal{N}}\left(1+\sqrt{1+4 \frac{\epsilon N_{i}}{h_{i}} \Omega_{0}}\right), \\
& q_{i} \approx \bar{Q} / n \text { for } i \in \mathcal{N},
\end{aligned}
$$

where $\Omega_{0}$ is given by (21).

The proof can be found in Appendix 9.9.

Proposition 8 Let the total jamming power $\bar{Q}$ be small. Then, the Nash equilibrium $(p, q)$ can be approximated as follows:

$$
\begin{gathered}
p_{i} \approx \frac{\sqrt{N_{i} / h_{i}}}{\sum_{j \in \mathcal{N}} \sqrt{N_{j} / h_{j}}} \bar{P} \text { for } i \in \mathcal{N} \\
q \in \mathcal{Q} \operatorname{such} \operatorname{that} \operatorname{supp}(q) \subset \mathcal{J},
\end{gathered}
$$


where $\Omega_{0}$ is given by (21), and

$$
\begin{aligned}
\operatorname{supp}(q) & \triangleq\left\{i \in \mathcal{N}: q_{i}>0\right\} \\
\mathcal{J} & \triangleq\left\{i \in \mathcal{N}: N_{i} h_{i} / g_{i}^{2}=\min _{j \in \mathcal{N}} N_{j} h_{j} / g_{j}^{2}\right\}
\end{aligned}
$$

The proof can be found in Appendix 9.10.

In particular, Proposition 7 and Proposition 8 imply that with large or small total jamming power resources, the jammer's strategy and the transmitter's strategy are insensitive to the TCC.

\subsection{Large or small threshold of communica- tion connectivity}

In this section we consider the cases where the TCC is either small or large.

Proposition 9 (a) Let the TCC $\epsilon$ be large. Then the Nash equilibrium $(p, q)$ can be approximated by (37) and (38) of Proposition 6.

(b) Let the TCC $\epsilon$ be small. Then, the Nash equilibrium $(p, q)$ can be approximated by (34) and (33) of Proposition 5 .

The proof can be found in Appendix 9.11.

\section{ALGORITHM TO ARRIVE AT THE EQUI- LIBRIUM}

In this section, an algorithm based on superposition of two bisection methods to arrive at equilibrium strategies is given.

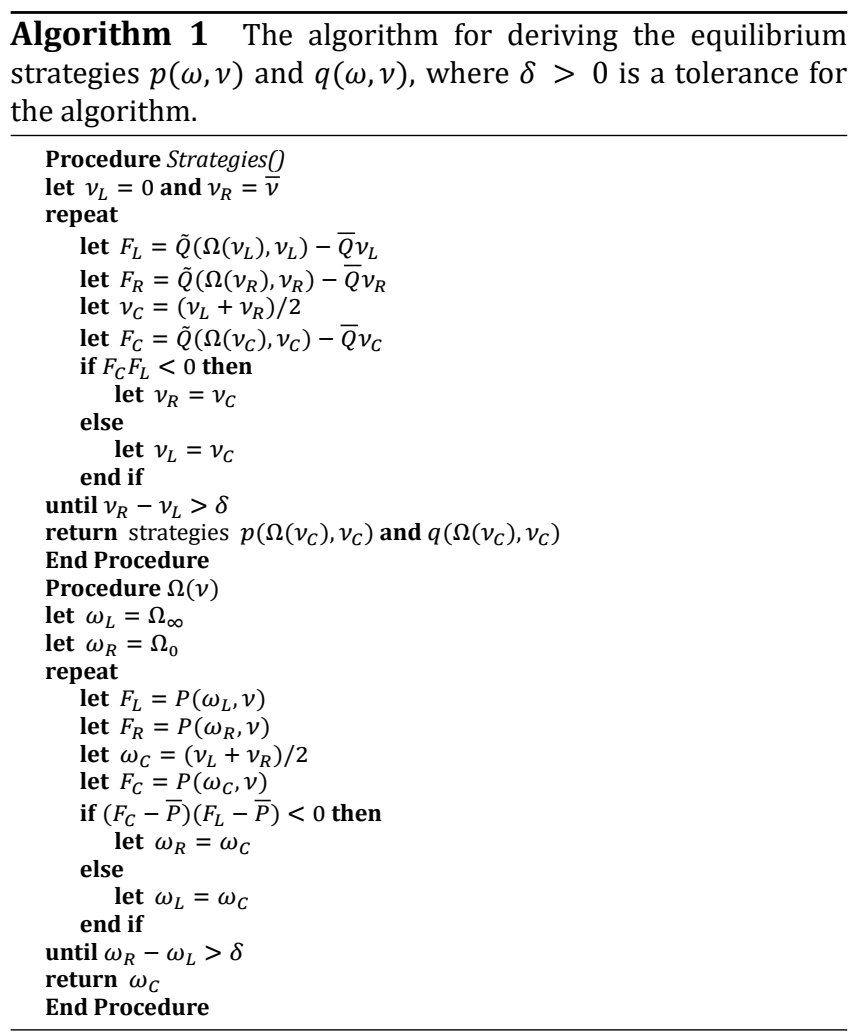

Proposition 10 Algorithm 1 converges to an equilibrium.

The proof can be found in Appendix 9.12.

The complexity involved in designing the equilibrium strategies by Algorithm 1 is $\log _{2}(\bar{v} / \delta) \log _{2}\left(\left(\Omega_{0}-\Omega_{\infty}\right) / \delta\right)$.

\section{DISCUSSION OF THE RESULTS}

In this section we illustrate Algorithm 1 using a system consisting of $n=5$ receivers, with fading gains from the transmitter to the receivers $h=(1,2,3,4,5)$, fading gains from the jammer to the receivers $g=(3,2,1,4,1)$, the background noises at the receivers $N=(3,2,1,4,1)$, the total transmitter power budget $\bar{P}=2$ and the total jamming power budget $\bar{Q}=3$. Fig. 1(a) illustrates that an increase in the total transmission power leads to an increase in the PCC (i.e., in $\pi$ ), while an increase in the jamming power reduces the PCC. Fig. 1(b) illustrates the transmitter's normalized strategies, i.e., $p / \bar{P}$, while Fig. 1(c) illustrates the jammer's strategies for the total power transmitter budget $\bar{P} \in\{0.1,1,10,100\}$. It shows that the jammer's strategy for a small total transmitter power budget $\bar{P}$ tends to a uniform strategy (Proposition 6), while for a large total transmitter power budget $\bar{P}$ the jammer's strategy tends to waterfilling-form strategies given by (33). Due to the waterfilling form of Equation (33), smaller $N_{i} h_{i} / g_{i}^{2}$ calls for applying larger jamming efforts. Here we have that

$$
N h / g^{2}=(0.3,1,3,1,5)
$$

For this reason, the largest jamming effort is focused on receiver 1 while receiver 2 and receiver 4 face approximately equal-level of interfering signals. Fig. 2(a) illustrates that an increase in the total jamming power leads to a decrease in the PCC, while an increase in the total transmission power reduces such negative effect from jammer's interference. Fig. 2(c) illustrates normalized jammer's strategies, i.e., $q / \bar{Q}$, while Fig. 2(b) illustrates transmitter's strategies for total jamming power budget $\bar{Q} \in\{0.1,1,10,100\}$. By (44) and (45), we have that $\mathcal{J}=\{1\}$. That is why, jamming efforts for small total power jamming budget $\bar{Q}$ is focused on receiver 1 (Proposition 8), while jamming efforts for large total jamming power budget $\bar{Q}$ tends to uniform distribution over all the receivers (Proposition 7). Fig. 3(a) illustrates that an increase in the TCC $\epsilon$ leads to a decrease in the PCC, while Fig. 3(b) and Fig. 3(c) illustrate that the jammer's strategy focuses jamming efforts on receiver 1 due to a waterfilling form of Equation (33) and (45) for a small TCC. For a large TCC, jammer's strategy tends to a uniform one (Proposition 9). In all of the cases, the transmitter tries to communicate with each receiver (i.e., $p_{i}>0$ for all i). This observation makes the MLCC problem remarkably different from standard OFDM communication scenarios where the transmitter, lacking sufficient transmission resources, must avoid transmission in some of the channels. 


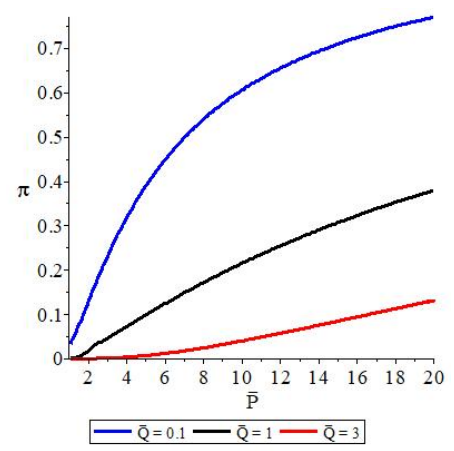

(a)

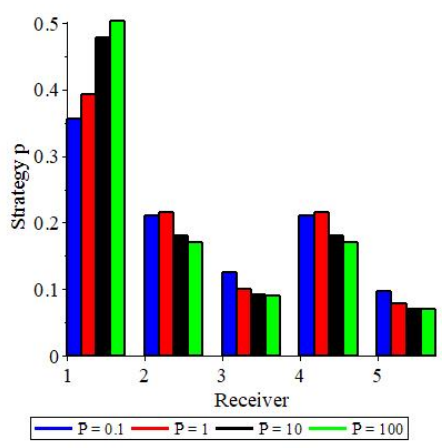

(b)

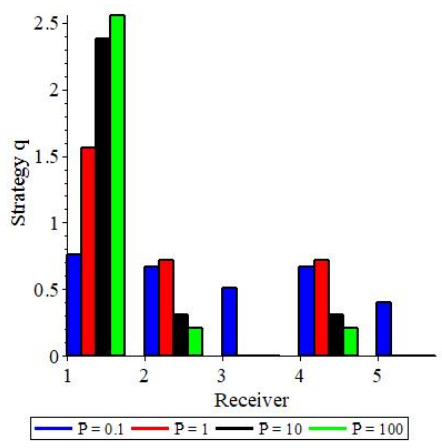

(c)

Fig. 1 - (a) The PCC $\pi$, (b) normalized transmitter's strategy $p$ with $\bar{Q}=3$ and (c) jammer's strategy $q$ with $\bar{Q}=3$ as functions of $\bar{P}$.

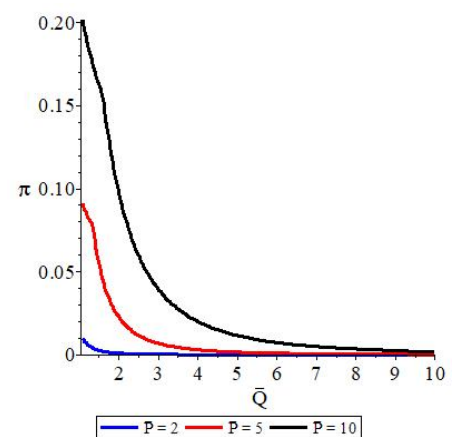

(a)

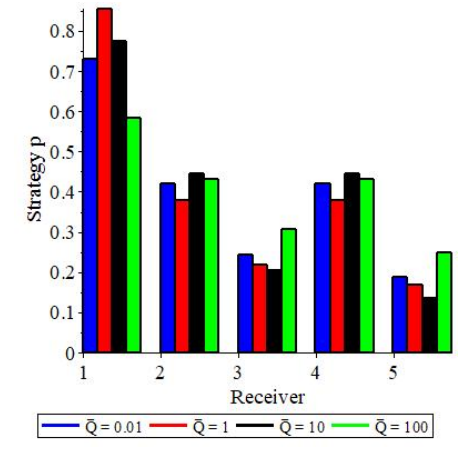

(b)

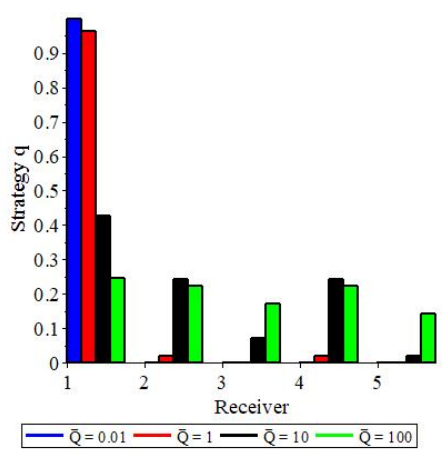

(c)

Fig. 2 - (a) The PCC $\pi$, (b) transmitter's strategy $p$ and (c) normalized jammer's strategy $q$ as functions of $\bar{Q}$.

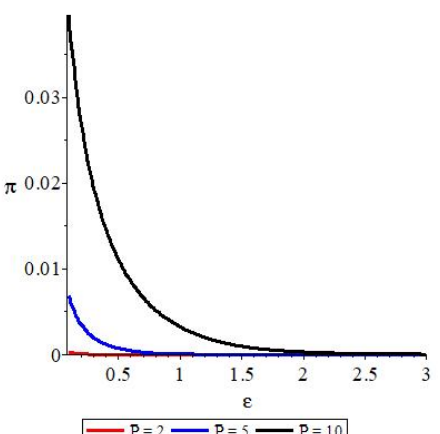

(a)

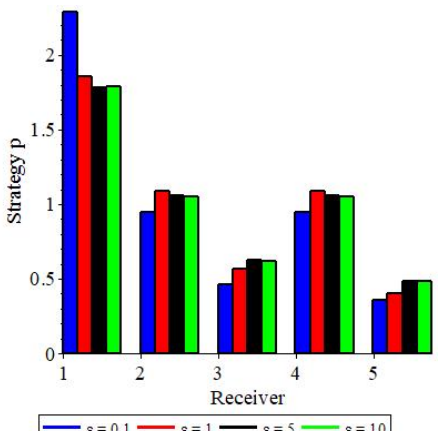

(b)

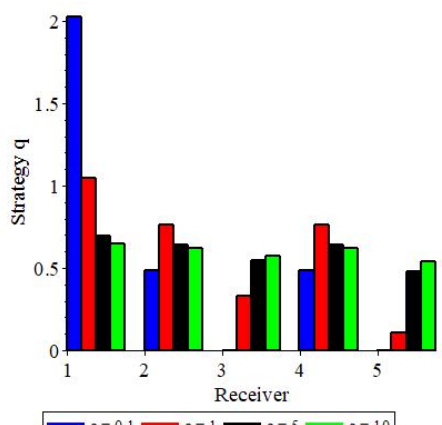

(c)

Fig. 3 - (a) The PCC $\pi$, (b) normalized transmitter's strategy $p$ with $\bar{Q}=3$ and (c) jammer's strategy $q$ with $\bar{Q}=3$ as functions of $\bar{P}$. 
Also, in OFDM communication, the transmitter's strategy becomes uniform in a high SINR mode, i.e., large transmission power, [25], while in the MLCC problem the jammer' strategy tends to a uniform one if either the total jamming resource budget is large, or the total transmission resource budget is small.

\section{CONCLUSIONS}

A problem of multi-link communication connectivity under jamming of a transmitter with a group of receivers when the channels are affected by Rayleigh fading has been formulated as a zero-sum power resource allocation game. Existence and uniqueness of the equilibrium in power allocation strategies have been proven. Thus, in contrast to Colonel Blotto games, if channels are affected by Rayleigh fading, then the stability of communication connectivity in a multi-link system can be maintained without introducing a random factor for a decision maker. Also, the problem of designing the equilibrium power allocation strategies has been reduced to the problem of finding a fixed point of a real-valued function. An algorithm based on the bisection method for finding the fixed point has been developed and its convergence has been proven.

\section{ACKNOWLEDGEMENT}

This work was supported in part by the U.S. National Science Foundation under Grant ACI-1541069, Grant CCF1908308 and Grant CNS-1909186.

\section{APPENDIX}

\subsection{Proof of Theorem 1}

Note that $v(p, q)$ is an additively separable function of $\left(p_{i}, q_{i}\right), i \in \mathcal{N}$ and

$$
\frac{\partial v^{2}(p, q)}{\partial p_{i}^{2}}=-\frac{\epsilon q_{i} g_{i}\left(\epsilon g_{i} q_{i}+2 h_{i} p_{i}\right)}{\left(\epsilon g_{i} q_{i}+h_{i} p_{i}\right)^{2} p_{i}^{2}}-\frac{2 \epsilon N_{i}}{h_{i} p_{i}^{3}}<0
$$

and

$$
\frac{\partial v^{2}(p, q)}{\partial q_{i}^{2}}=\frac{\epsilon^{2} g_{i}^{2}}{\left(\epsilon g_{i} q_{i}+h_{i} p_{i}\right)^{2}}>0 .
$$

Thus, $v(p, q)$ is concave in $p$ and convex in $q$, and the result follows from the Nash's theorem [2] since sets $\mathcal{P}$ and $\mathcal{Q}$ of feasible strategies of the transmitter and the jammer are compact.

\subsection{Proof of Proposition 1}

By (46), the NLP problem (12) is a concave problem. Thus, to solve the NLP (12) we introduce Lagrangian $\mathcal{L}_{T, \omega}(p)$ with $\omega$ is a Lagrange multiplier: $\mathcal{L}_{T, \omega}(p)=v(p, q)+$ $\omega\left(\bar{P}-\sum_{i=1}^{n} p_{i}\right)$. Then, for a fixed $q \in \mathcal{Q}$, following [21] and the KKT Theorem, $p \in \mathcal{P}$ is the best response if and only if the following condition holds:

$$
\frac{\partial \mathcal{L}_{T, \omega}(p)}{\partial p_{i}}=\frac{\epsilon g_{i} q_{i}}{\left(\epsilon g_{i} q_{i}+h_{i} p_{i}\right) p_{i}}+\frac{\epsilon N_{i}}{h_{i} p_{i}^{2}}-\omega \begin{cases}=0, & p_{i}>0 \\ \leq 0, & p_{i}=0 .\end{cases}
$$

By (47), the NLP (13) is a convex problem. Thus, to solve the NLP (13) we introduce Lagrangian $\mathcal{L}_{I, v}(q)$ with $v$ is a Lagrange multiplier as follows: $\mathcal{L}_{J, v}(q)=-v(p, q)+$ $v\left(\bar{Q}-\sum_{i=1}^{n} q_{i}\right)$. Then, similarly, for a fixed $p \in \mathcal{P}, q \in \mathcal{Q}$ is the best response if and only if the following condition holds:

$$
\frac{\partial \mathcal{L}_{J, v}(q)}{\partial q_{i}}=\frac{\epsilon g_{i}}{\epsilon g_{i} q_{i}+h_{i} p_{i}}-v \begin{cases}=0, & q_{i}>0 \\ \leq 0, & q_{i}=0\end{cases}
$$

By (48), we have that

$$
p_{i}>0 \text { for any } i \text {. }
$$

Then, by (48) and (49), $\omega>0$ and $v>0$ correspondingly. By (48), only two cases arise to consider: (I) $p_{i}>0, q_{i}=0$ and (II) $p_{i}>0, q_{i}>0$.

(I) Let $p_{i}>0$ and $q_{i}=0$. Then, by (48),

$$
p_{i}=\sqrt{\frac{\epsilon N_{i}}{h_{i} \omega}}
$$

Substituting (51) into (49) implies

$$
\frac{\epsilon g_{i}^{2}}{N_{i} h_{i}} \omega \leq v^{2}
$$

(II) Let $p_{i}>0$ and $q_{i}>0$. Then, by (48) and (49), we have that

$$
\frac{\epsilon g_{i} q_{i}}{\left(\epsilon g_{i} q_{i}+h_{i} p_{i}\right) p_{i}}+\frac{\epsilon N_{i}}{h_{i} p_{i}^{2}}=\omega
$$

and

$$
\epsilon g_{i} q_{i}+h_{i} p_{i}=\frac{\epsilon g_{i}}{v}
$$

Thus,

$$
q_{i}=\frac{1}{v}-\frac{h_{i}}{\epsilon g_{i}} p_{i} .
$$

Substituting (55) into (53) implies

$$
\frac{1}{p_{i}}+\frac{\epsilon N_{i}}{h_{i} p_{i}^{2}}=\omega+\frac{h_{i}}{\epsilon g_{i}} \nu .
$$

Solving (56) on $p_{i}$ implies that

$$
p_{i}=\frac{1+\sqrt{1+4 \frac{\epsilon N_{i}}{h_{i}}\left(\omega+\frac{h_{i}}{\epsilon g_{i}} v\right)}}{2\left(\omega+\frac{h_{i}}{\epsilon g_{i}} v\right)} .
$$

Thus,

$$
q_{i}=\frac{1}{v}-\frac{h_{i}}{\epsilon g_{i}} \frac{1+\sqrt{1+4 \frac{\epsilon N_{i}}{h_{i}}\left(\omega+\frac{h_{i}}{\epsilon g_{i}} v\right)}}{2\left(\omega+\frac{h_{i}}{\epsilon g_{i}} v\right)} .
$$


By (57), $p_{i}>0$. By (58), $q_{i}>0$ if and only if

$$
\frac{2}{v}\left(\omega+\frac{h_{i}}{\epsilon g_{i}} v\right)>\frac{h_{i}}{\epsilon g_{i}}\left(1+\sqrt{1+4 \frac{\epsilon N_{i}}{h_{i}}\left(\omega+\frac{h_{i}}{\epsilon g_{i}} v\right)}\right) .
$$

The last inequality is equivalent to

$$
\frac{1}{v}\left(2 \omega+\frac{h_{i}}{\epsilon g_{i}} v\right)>\frac{h_{i}}{\epsilon g_{i}} \sqrt{1+4 \frac{\epsilon N_{i}}{h_{i}}\left(\omega+\frac{h_{i}}{\epsilon g_{i}} v\right)} .
$$

A straightforward calculation shows that (59) is equivalent to

$$
\frac{\epsilon g_{i}^{2}}{N_{i} h_{i}} \omega>v^{2}
$$

Thus, $q_{i}$, given by (58), is positive if and only if (60) holds. Finally, combining (I) and (II) implies the result.

\subsection{Proof of Proposition 2}

A straightforward substituting $\frac{\epsilon g_{i}^{2}}{N_{i} h_{i}} \omega=v^{2}$ into (14) implies (a).

Let

$$
\eta(x)=\frac{1+\sqrt{1+a x}}{x}=\frac{1}{x}+\sqrt{\frac{1}{x^{2}}+\frac{a}{x}},
$$

where $a$ is a positive parameter. It is clear that

$$
\eta(x) \text { is decreasing in } x>0 .
$$

Let

$$
x=\omega+\frac{h_{i}}{\epsilon g_{i}} v \text { and } a=4 \frac{\epsilon N_{i}}{h_{i}} .
$$

Substituting (63) into (61), by (14) and (62), yields (b) and (c). (d) follows from (b). (e) follows from (a) and (b), while (21) and (22) follow from (18), (19) and (20).

\subsection{Proof of Proposition 3}

(a) follows from (25).

Let

$$
f(v)=\frac{v}{c+a v}+\frac{\sqrt{1+b(c+a v)}}{c+a v},
$$

where $a=h_{i} /\left(\epsilon g_{i}\right), b=4 \epsilon N_{i} / h_{i}$ and $c=\omega$. Then

$\frac{d f(v)}{d v}=\frac{c}{(c+a v)^{2}}+\frac{\left(a^{2} b v^{2}+3 a b c v+2 b c^{2}+2 c\right)}{2(a v+c)^{2} \sqrt{a b v+b c+1}}>0$.

Now we establish that

$$
\tilde{Q}(\omega, v)=0 \text { for } v \geq \bar{v} \text { and } \omega \in\left[\Omega_{\infty}, \Omega_{0}\right]
$$

By (25), $\tilde{Q}(\omega, v)=0$ if and only if for all $i$ the following inequality holds:

$$
2\left(\omega+\frac{h_{i}}{\epsilon g_{i}} v\right) \leq \frac{h_{i}}{\epsilon g_{i}} v\left(1+\sqrt{1+4 \frac{\epsilon N_{i}}{h_{i}}\left(\omega+\frac{h_{i}}{\epsilon g_{i}} v\right)}\right)
$$

Since $\omega \geq \Omega_{\infty}>0$, substituting $\omega=\Omega_{\infty}$ into the rightside of (67) implies that if the following inequality holds then (67) also holds:

$$
2\left(\omega+\frac{h_{i}}{\epsilon g_{i}} v\right) \leq \frac{h_{i}}{\epsilon g_{i}} v \sqrt{4 \frac{\epsilon N_{i}}{h_{i}} \frac{h_{i}}{\epsilon g_{i}} v}
$$

Taking into account notations (27) and that $\omega \leq \Omega_{0}$, (68) holds for all $i$ if the following inequality holds:

$$
A+B v \leq C v^{3 / 2}
$$

with $A, B$ and $C$ given by (27). It is clear that Equation (26) has the unique positive root $v=\bar{v}$. Then, (69) holds for $v \geq \bar{v}$, and (66) follows.

Thus, (25), (64), (65) and (66) imply (b). (c) follows from (25) and (61)-(63). Also, (b), (c), (66) and Proposition 2(e) imply (d).

Since the right-side of Equation (23) is increasing from zero for $v=0$ to infinity for $v \uparrow \infty$ while, by (d), the rightside of Equation (23) is decreasing and reaches zero for $v \geq \bar{v}$, (e) follows.

\subsection{Proof of Theorem 2}

By Proposition 1, all the equilibrium strategies have to have the form given by (14) and (15), where $(\omega, v)$ is a positive solution of (16) and (17). By Proposition 2, (14) establishes a bijection relation between $\omega$ and $v$ given by function $\omega=\Omega(v)$. Substituting this function into (15) yields into Equation (23) of one variable $v$. By Proposition 3 , this equation has the unique root. Thus, the equilibrium also is unique, and the result follows.

\subsection{Proof of Proposition 4}

By (30), (52) cannot hold for any $i$. Thus, by (14) and (15), $p_{i}>0$ and $q_{i}>0$ for any $i$. Substituting (30) into (14) and (15) implies

$$
\begin{aligned}
& p_{i}(\omega, v)=\frac{1}{\omega+v h_{i} /\left(\epsilon g_{i}\right)}, \\
& q_{i}(\omega, v)=\frac{\omega / v}{\omega+v h_{i} /\left(\epsilon g_{i}\right)} \text { for any } i .
\end{aligned}
$$

Dividing (71) by (70) implies that

$$
q_{i}(\omega, v)=(\omega / v) p_{i}(\omega, v)
$$

Summing up (72) by $i$ and taking into account that $p \in \mathcal{P}$ and $q \in \mathcal{Q}$ we have that

$$
\bar{Q}=(\omega / v) \bar{P} .
$$


Thus, $v=\bar{P} \omega / \bar{Q}$. Substituting this $v$ into (70) implies that

$$
p_{i}(\omega, v)=1 /\left(\omega\left(1+h_{i} \bar{P} /\left(\epsilon g_{i} \bar{Q}\right)\right)\right) .
$$

Since $p \in \mathcal{P}$, summing up (74) yields that $\omega=$ $\sum_{i \in \mathcal{N}} 1 /\left(\bar{P}\left(1+h_{i} \bar{P} /\left(\epsilon g_{i} \bar{Q}\right)\right)\right)$. This and (74) yield (31), while (32) follows from (31) and (72).

\subsection{Proof of Proposition 5}

Since $\bar{P} \uparrow \infty$ there is at least one $i$ such $p_{i} \uparrow \infty$. Then, by (54), $v \downarrow 0$ and for large $\bar{P}$ we have that

$$
p_{i} \approx \epsilon g_{i} /\left(h_{i} v\right) .
$$

By (56), for large $p_{i}$ we have that

$$
1 / p_{i} \approx \omega+\left(h_{i} / \epsilon g_{i}\right) v .
$$

Thus, $v \downarrow 0$ while $\bar{P} \uparrow \infty$. Moreover, by (75) and (76),

$$
\omega / v \downarrow 0 \text { for } \bar{P} \uparrow \infty .
$$

Then, since $\sqrt{1+a x} \approx 1+a x / 2$ for small $x$, (15) implies that

$$
\begin{aligned}
q_{i} & \approx\left\lfloor\frac{1}{v}-\frac{h_{i}}{\epsilon g_{i}} \frac{2+2 \frac{\epsilon N_{i}}{h_{i}}\left(\omega+\frac{h_{i}}{\epsilon g_{i}} v\right)}{2\left(\omega+\frac{h_{i}}{\epsilon g_{i}} v\right)}\right\rfloor_{+} \\
& =\left\lfloor\frac{\omega}{v^{2}\left(\omega / v+h_{i} /\left(\epsilon g_{i}\right)\right)}-\frac{N_{i}}{g_{i}}\right\rfloor_{+} .
\end{aligned}
$$

By (77) and (78), we have that

$$
\left.q_{i} \approx \frac{g_{i}}{h_{i}} \mid \frac{\epsilon \omega}{v^{2}}-\frac{N_{i} h_{i}}{g_{i}^{2}}\right]_{+} .
$$

Let $\tau=\epsilon \omega / \nu^{2}$. Substituting this $\tau$ into (79) and taking into account that $q \in Q$ imply (33) and (35).

By (14) and (75), we have that

$$
p_{i} \approx \begin{cases}\sqrt{\frac{\epsilon N_{i}}{h_{i} \omega}}, & \tau \leq \frac{N_{i} h_{i}}{g_{i}^{2}}, \\ \frac{\epsilon g_{i}}{v h_{i}}, & \tau>\frac{N_{i} h_{i}}{g_{i}^{2}} .\end{cases}
$$

Summing up (80) and taking into account that $p \in \mathcal{P}$ yields the following relation: $\bar{P}=$ $\sqrt{\frac{\epsilon}{\omega}}\left(\sum_{\tau \leq N_{i} h_{i} / g_{i}^{2}} \sqrt{\frac{N_{i}}{h_{i}}}+\sqrt{\tau} \sum_{\tau>N_{i} h_{i} / g_{i}^{2}} \frac{g_{i}}{h_{i}}\right)$. This allows to define $\sqrt{\epsilon / \omega}$. Substituting this $\sqrt{\epsilon / \omega}$ into (80) implies (34) and (36), and the result follows.

\subsection{Proof of Proposition 6}

Note that $p_{i} \downarrow 0$ since $\bar{P} \downarrow 0$. Then, by (53),

$$
\omega \uparrow \infty .
$$

Since $q \in \mathcal{Q}$, by (55), $v$ is upper-bounded on $\omega$. This, (14) and (15) imply that $q_{i}>0$ for any $i$. Then, by (55),

$$
q_{i} \approx 1 / v \text { for all } i \text { and small } \bar{P} .
$$

Since $q \in \mathcal{Q}$, summing up (82) by $i \in \mathcal{N}$ implies that

$$
v \approx n / \bar{Q} \text {. }
$$

Then, (82) and (83) imply (38).

Substituting (83) into (53), by (81), implies that for small $\bar{P}$ the following approximation holds:

$$
p_{i} \approx \sqrt{\epsilon N_{i} /\left(h_{i} \omega\right)}
$$

This and the fact that $p \in \mathcal{P}$ implies (37).

\subsection{Proof of Proposition 7}

Since $q \in \mathcal{Q}, \bar{Q} \uparrow \infty$ implies that $q_{i} \uparrow \infty$ for at least one $i$. Then, by (55), $v \downarrow 0$. Thus, by (55), for large $\bar{Q}$ we have that

$$
q_{i} \approx v \text { for all } i \text {. }
$$

Taking into account that $q \in \mathcal{Q}$ summing up (85) yields that $v=\bar{Q} / n$. Substituting this $v$ into (85) implies (40). While substituting $v=\bar{Q} / n$ into (14) and taking into account that $\bar{Q} \uparrow \infty$ imply (39), and the result follows.

\subsection{Proof of Proposition 8}

First prove that $v$ cannot tend to infinity while $\bar{Q}$ tends to zero. Assume that $v \uparrow \infty$. Then, by (55), $p_{i} \downarrow 0$ where $q_{i}>0$. Thus, by (53), $\omega \uparrow \infty$. So, by (14), $p_{i} \downarrow 0$ also for $q_{i}=0$. This contradicts the fact that $p \in \mathcal{P}$. Thus, $v$ cannot tend to infinity while $\bar{Q} \downarrow 0$. Then, since $q_{i} \downarrow 0$ while $\bar{Q} \downarrow 0$, by (55), we have that

$$
v \approx \epsilon g_{i} /\left(h_{i} p_{i}\right) \text { for } q_{i}>0 .
$$

While, by (53), since $q_{i} \downarrow 0$, we have that,

$$
p_{i} \approx \sqrt{\epsilon N_{i} /\left(h_{i} \omega\right)} \text { for } q_{i}>0 .
$$

By (14), $p_{i}=\sqrt{\epsilon N_{i} /\left(h_{i} \omega\right)}$ for $q_{i}=0$. This, (87) and the fact that $p \in \mathcal{P}$ implies (41).

Substituting (87) into (86) implies (42), and the result follows.

\subsection{Proof of Proposition 9}

By (8), since $p \in \mathcal{P}$, the equilibrium strategies are defined by ratio $\bar{P} / \epsilon$, and the result follows. 


\subsection{Proof of Proposition 10}

First note that procedure $\Omega(v)$ reflects the bisection method to solve equation $P(\omega, v)=\bar{P}$ for a fixed $v$. By Proposition 2, this procedure converges. Procedure Strategies() reflects the bisection method to solve equation $\tilde{Q}(\Omega(v), v)-\bar{Q} v=0$. By Proposition 3, this procedure also converges. Thus, Algorithm 1 converges as a superposition of two converging procedures.

\section{REFERENCES}

[1] S. Vadlamania, B. Eksioglub, H. Medala, and A. Nandia. "Jamming attacks on wireless networks: A taxonomic survey". In: International Journal of Production Economics 172 (2016), pp. 76-94.

[2] Z. Han, D. Niyato, W. Saad, T. Basar, and A. Hjungnes. Game Theory in Wireless and Communication Networks: Theory, Models, and Applications. Cambridge University Press, 2012.

[3] D. Yang, G. Xue, J. Zhang, A. Richa, and X. Fang. “Coping with a Smart Jammer in Wireless Networks: A Stackelberg Game Approach". In: IEEE Transactions on Wireless Communications 12 (2013), pp. 40384047.

[4] A. Garnaev, A. Petropulu, W. Trappe, and H.V Poor. "A Jamming Game with Rival-Type Uncertainty". In: IEEE Transactions on Wireless Communication 19 (2020), pp. 5359-5372.

[5] F.M Aziz, J.S Shamma, and G.L Stuber. "Jammer-type estimation in LTE with a smart jammer repeated game". In: IEEE Transactions on Vehicular Technology 66 (2017), pp. 7422-7431.

[6] T. Song, W.E Stark, T. Li, and J.K Tugnait. “Optimal Multiband Transmission Under Hostile Jamming". In: IEEE Transactions on Communications 64 (2016), pp. 4013-4027.

[7] L. Xiao, D. Jiang, D. Xu, H. Zhu, Y. Zhang, and H.V Poor. "Two-Dimensional Antijamming Mobile Communication Based on Reinforcement Learning". In: IEEE Transactions on Vehicular Technology 67 (2018), pp. 9499-9512.

[8] N. Abuzainab and W. Saad. "Dynamic Connectivity Game for Adversarial Internet of Battlefield Things Systems". In: IEEE Internet of Things Journal 5 (2018), pp. 378-390.

[9] Y. Gao, Y. Xiao, M. Wu, M. Xiao, and J. Shao. "Game Theory-Based Anti-Jamming Strategies for Frequency Hopping Wireless Communications". In: IEEE Transactions on Wireless Communications 17 (2018), pp. 5314-5326.

[10] A. Garnaev, A. Petropulu, W. Trappe, and H.V Poor. "A Muli-Jammer Game with Latency as the User's Communication Utility". In: IEEE Communications Letters 24 (2020), pp. 1899-1903.
[11] A. Garnaev, A. Petropulu, W. Trappe, and H.V Poor. "A Power Control Game with Uncertainty On the Type of the Jammer". In: Proc. IEEE Global Conference on Signal and Information Processing (GlobalSIP). 2019.

[12] N. Namvar, W. Saad, N. Bahadori, and B. Kelleys. "Jamming in the Internet of Things: A GameTheoretic Perspective". In: Proc. IEEE Global Communications Conference (GLOBECOM). 2016.

[13] Y. Wu, B. Wang, and K.J.R Liu. "Optimal power allocation strategy against jamming attacks using the Colonel Blotto game". In: Proc. IEEE Global Telecommunications Conference (GLOBECOM). 2009, pp.15.

[14] G.D Nguyen, S. Kompella, C. Kam, J.E Wieselthier, and A. Ephremides. "Wireless link connectivity under hostile interference: Nash and Stackelberg equilibria". In: Proc. 14th International Symposium on Modeling and Optimization in Mobile, $\mathrm{Ad}$ Hoc, and Wireless Networks (WiOpt). 2016.

[15] G.D Nguyen, S. Kompella, C. Kam, J.E Wieselthier, and A. Ephremides. "Impact of Hostile Interference on Wireless Link Connectivity". In: IEEE Transactions on Control of Network Systems 5 (2018), pp. 1445-1456.

[16] R. El-Bardan, S. Brahma, and P.K Varshney. "Power control with jammer location uncertainty: A game theoretic perspective". In: Proc. 48th Annual Conference on Information Sciences and Systems (CISS). 2014, pp. 1-6.

[17] A. Garnaev and W. Trappe. "Stability of communication link connectivity against hostile interference". In: Proc. IEEE Global Conference on Signal and Information Processing (GlobalSIP). 2017.

[18] M. Hajimirsaadeghi and N.B Mandayam. "A dynamic Colonel Blotto game model for spectrum sharing in wireless networks". In: Proc. 55th Annual Allerton Conference on Communication, Control, and Computing (Allerton). 2017, pp. 287-294.

[19] M. Labib, S. Ha, W. Saad, and J.H Reed. "A Colonel Blotto Game for Anti-Jamming in the Internet of Things". In: Proc. IEEE Global Communications Conference (GLOBECOM). 2015, pp. 1-6.

[20] C. Guo, Y. Zhang, M. Sheng, Wang X, and Y. Li. " $\alpha$-Fair Power Allocation in Spectrum-Sharing Networks". In: IEEE Transactions on Vehicular Technology 65 (2016), pp. 3771-3777.

[21] E. Altman, K. Avrachenkov, and A. Garnaev. "Fair Resource Allocation in Wireless Networks in the Presence of a Jammer". In: Performance Evaluation 67 (2010), pp. 338-349.

[22] E. Altman, K. Avrachenkov, and A. Garnaev. "Closed Form Solutions for Water-Filling Problem in Optimization and Game Frameworks". In: Telecommunication Systems Journal 47 (2011), pp. 153-164. 
[23] V. Baston and A. Garnaev. "A Search Game with a Protector". In: Naval Research Logistics 47 (2000), pp. 85-96.

[24] E. Altman, K. Avrachenkov, and A. Garnaev. "Jamming Game in Wireless Networks with Transmission Cost". In: Network Control and Optimization. Ed. by T. Chahed and B. Tuffin. Vol. 4465. LNCS. Springer, 2007, pp. 1-12.

[25] A. Garnaev, W. Trappe, and A.Petropulu. "Equilibrium Strategies for an OFDM Network That Might be Under a Jamming Attack". In: Proc. 51st Annual Conference on Information Systems and Sciences (CISS). 2017, pp. 1-6.

\section{AUTHORS}

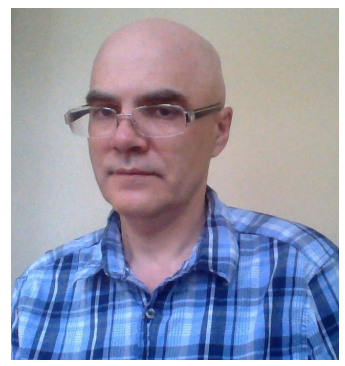

Andrey Garnaev received a Ph.D. degree in applied mathematics and D.Sc. degree in computer science and applied mathematics from Saint Petersburg State University, St. Petersburg, Russia, in 1987 and 1997, respectively. He is currently with WINLAB, Rutgers University, USA. His current research interests are in applications of game theory and optimization theory in network security, wireless communication and related fields.

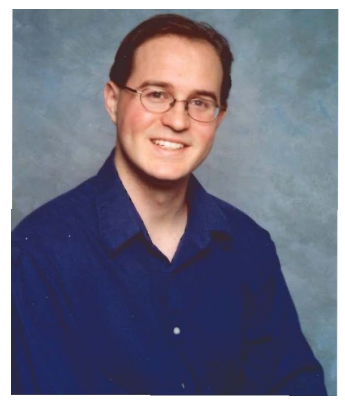

Wade Trappe is a Professor in the Electrical and Computer Engineering Department at Rutgers University, and Associate Director of the Wireless Information Network Laboratory (WINLAB), where he directs WINLAB's research in wireless security. He has led several federally funded projects in the area of cybersecurity and communication systems, projects involving security and privacy for sensor networks, physical layer security for wireless systems, a security framework for cognitive radios, the development of wireless testbed resources (the ORBIT testbed, www.orbit-lab.org), and new RFID technologies. His experience in network security and wireless spans over 15 years, and he has co-authored a popular textbook in security, Introduction to Cryptography with Coding Theory, as well as several monographs on wireless security, including Securing Wireless Communications at the Physical Layer and Securing Emerging Wireless Systems: Lower-layer Approaches. Professor Trappe served as an editor for IEEE Transactions on Information Forensics and Security (TIFS), IEEE Signal Processing Magazine (SPM), and IEEE Transactions on Mobile Computing (TMC). He served as the IEEE Signal Processing Society representative to the governing board of IEEE TMC. Professor Trappe is an IEEE Fellow.

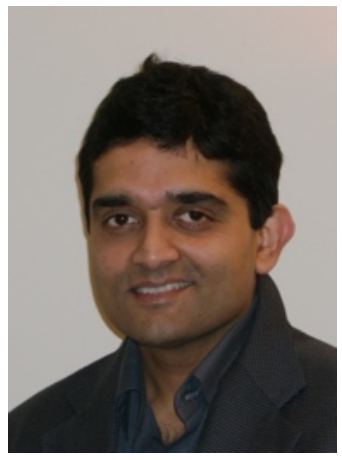

Narayan B. Mandayam is a Distinguished Professor and Chair of Electrical and Computer Engineering at Rutgers University, where he also serves as Associate Director of WINLAB. Using constructs from game theory, communications, and networking, his work has focused on system modeling, information processing as well as resource management. His recent interests include enabling privacy in IoT, building resilience in smart city infrastructures and trustworthy knowledge creation on the Internet. He received the 2015 IEEE COMSOC Advances in Communications Award, the 2014 IEEE Donald G. Fink Award, and the 2009 Fred W. Ellersick Prize from the IEEE Communications Society. He is also a recipient of the Peter D. Cherasia Faculty Scholar Award from Rutgers University (2010), the National Science Foundation CAREER Award (1998), the Institute Silver Medal from the Indian Institute of Technology (1989), Kharagpur and its Distinguished Alumnus Award (2018). He is a Fellow and Distinguished Lecturer of the IEEE.

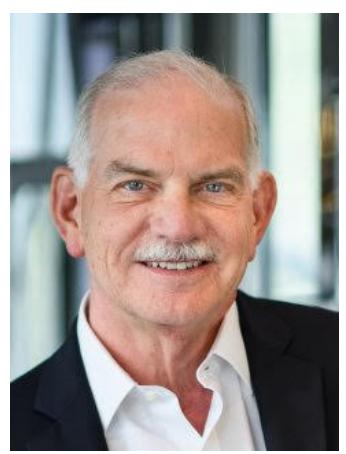

H. Vincent Poor $\left(S^{\prime} 72, M^{\prime} 77\right.$, SM'82, F'87) received a Ph.D. degree in EECS from Princeton University in 1977. From 1977 until 1990, he was on the faculty of the University of Illinois at Urbana-Champaign. Since 1990 he has been on the faculty at Princeton, where he is currently the Michael Henry Strater University Professor. During 2006 to 2016, he served as the dean of Princeton?s School of Engineering and Applied Science. He has also held visiting appointments at several other universities, including most recently at Berkeley and Cambridge. His research interests are in the areas of information theory, machine learning and network science, and their applications in wireless networks, energy systems and related fields. Among his publications in these areas is the forthcoming book Machine Learning and Wireless Communications (Cambridge University Press, 2021). Dr. Poor is a member of the National Academy of Engineering and the National Academy of Sciences, and is a foreign member of the Chinese Academy of Sciences, the Royal Society, and other national and international academies. Recent recognition of his work includes the 2017 IEEE Alexander Graham Bell Medal and a D.Eng. honoris causa from the University of Waterloo awarded in 2019. 